九州大学学術情報リポジトリ

Kyushu University Institutional Repository

\title{
ZERO-SUM GAMES WITH STOPPING TIMES AND VARIATIONAL INEQUALITIES
}

Yoshida, Yuj i

Department of Mathematics, Faculty of Science, Kyushu University

https://doi.org/10.5109/13401

出版情報: Bulletin of informatics and cybernetics. 23 (3/4), pp.141-154, 1989-03. Research Association of Statistical Sciences

バージョン:

権利関係 : 


\title{
ZERO-SUM GAMES WITH STOPPING TIMES AND VARIATIONAL INEQUALITIES
}

\author{
By
}

\section{Yuji YoshIDA*}

\begin{abstract}
This paper deals with a zero-sum game, whose state changes correspondingly with a kind of Markov process. In the game the two players' strategies are when to stop the game to maximize their own rewards.

In this paper we investigate optimal stopping times, optimal rewards and the conditions which should be satisfied. To describe more precisely, it is as follows.

Let $M$ be an $m$-symmetric Hunt process and let $(\mathfrak{F}, \mathfrak{F})$ be the corresponding Dirichlet space. For $\alpha>0$ and $W \in \mathfrak{F}$ we consider a variational inequality :

$$
U^{\alpha} \in \Re, \quad\left(\xi_{\alpha}\left(U^{\alpha}-W, V-U^{\alpha}\right) \geqq 0 \quad \text { for all } V \in \Re,\right.
$$

where $\Re$ is a certain subset of $\mathfrak{F}$. In this paper we investigate properties of solutions $U^{\alpha}$ of (1.1) and we show that solutions $U^{\alpha}$ has a quasi-continuous version which becomes to be a value of a certain zero-sum game associated with regions $X-F_{i}(i=1,2)$ not to permit stopping the game. The aim of this paper is to discuss these in the case of $m$-symmetric Hunt processes, which have $m$-symmetric diffusion processes and $m$-symmetric jump processes as examples. When the processes are transient, we can treat the case of $\alpha=0$ and we discuss it similarly.
\end{abstract}

\section{Introduction}

Zero-sum Markov games with stopping times are studied by several authors. N. V. Krylov [6] and L. Stettner [8] have studied them by making use of penalty methods. J. M. Bismut [2] has proved that a zero-sum game possesses a value from the standpoint of convex analysis. Especially A. Bensoussan and A. Friedman [1] and A. Friedman [4] have investigated a relation between a zero-sum Markov game with stopping times and a certain variational inequality in the case of diffusion processes. On the other hand H. Nagai [7] has used Dirichlet space to discuss a relation between a stopping problem and a variational inequality. Moreover J. Zabczyk [9] has discussed zero-sum Markov games in this situation. We deal with them in the case of an $m$ symmetric Hunt process and the corresponding regular Dirichlet space (FF, (F) by a different way from [9] and also discuss them in the transient case.

\footnotetext{
* Department of Mathematics, Faculty of Science, Kyushu University, Fukuoka, Japan.
} 
In the latter part of this section we state the notations of an $m$-symmetric process and the related results. In Section 1 we investigate a zero-sum game with a discounted rate $\alpha$ in the case of an $m$-symmetric Hunt process with a general regular Dirichlet space. In Section 2 we discuss a non-discounted zero-sum game in the case of an $m$ symmetric Hunt process with a transient regular Dirichlet space. In Section 3 we consider the case of an $m$-symmetric jump process as an example of Section 1 and we also consider the case of a diffusion in a bounded domain as an example of Section 2.

Let $X$ be a locally compact separable Hausdorff space and let $m$ be a positive Radon measure on $X$. Let $M=\left\{\Omega, \mathfrak{M}, \mathfrak{M}_{t}, X_{t}, P_{x}, \theta_{t}, \zeta\right\}$ be an $m$-symmetric Hunt process on $X$ and let $P_{t}$ and $R_{\alpha}$ be its transition function and resolvent respectively. In this paper we use notations and definitions of M. Fukushima [5] concerning Dirichlet space corresponding to $m$-symmetric Hunt process $M . L^{2}(X ; m)$ denotes the real $L^{2}$ space with the inner product $(\cdot, \cdot)$. Let $T_{t}\left(G_{\alpha}\right)$ be the $L^{2}$-transition function $\left(L^{2}-\right.$ resolvent) induced from $P_{t}\left(R_{\alpha}\right.$ resp.). ( $(\mathfrak{F},(\mathfrak{F})$ denotes a Dirichlet space associated with the process $M$. In this paper we assume that $(\mathfrak{F}, \mathfrak{F})$ is regular. $\|\cdot\|_{\infty}$ denotes the essential supremum norm on $X$ and $d(\cdot, \cdot)$ expresses a metric on $X$. We express by $\left\{\widetilde{F}_{t}\right\}_{0 \leqq t \leqq \infty}$ the minimum completed admissible nondecreasing family of sub- $\sigma$-algebras of $\mathfrak{M}$.

Hence for $\alpha \geqq 0$ we define a continuous additive functional $A=\{A(t)\}_{t \geq 0}$ associated with a multiplicative functional $\left\{e^{-\alpha t}\right\}_{t \geqq 0}$ as follows:

$A(t, \omega)$ is a real valued function on $[0, \infty) \times \Omega$ which is $\left\{\mathfrak{F}_{t}\right\}$-adapted and for which there exist a set $\Lambda \in \mathcal{F}$ and an exceptional set $N$ such that $P_{x}(\Lambda)=1$ for each $x \in X-N$ and for which the following properties $(\mathrm{F} 1) \sim(\mathrm{F} 4)$ are satisfied for each $\omega \in \Lambda$ :

(F1) $A(t, \omega)$ is finite and continuous in $t \geqq 0$,

(F2) $A(0, \omega)=0$,

(F3) $A(t, \omega)=A(\zeta(\omega), \omega)$ for each $t \geqq \zeta(\omega)$,

(F4) $A(t+s, \omega)=A(t, \omega)+e^{-\alpha t} A\left(s, \theta_{t} \omega\right)$ for each $t, s \geqq 0$.

Especially we note the property $(\mathrm{F} 4)$, which is induced from [3], is different from additive functionals in [5]. For $\alpha \geqq 0$ we express by $\mathfrak{U}_{c}^{\alpha}$ the set of all continuous additive functionals associated with a multiplicative functional $\left\{e^{-\alpha t}\right\}_{t \geq 0}$.

$C_{0}(X)$ denotes the space of all continuous functions on $X$ with compact supports. We define $\langle\nu, U\rangle=\int_{X} U(x) \nu(d x)$ for $U \in \mathscr{F}$ and Radon measures $\nu$ on $X$ and we write by $\tilde{U}$ a quasi-continuous version of $U$ for $U \in \widetilde{F}$ in accordance with [5]. In this paper we also use the following notations:

$$
\begin{aligned}
& U \vee V=\max \{U, V\} \text { and } U \wedge V=\min \{U, V\} \quad \text { for } U, V \in \mathfrak{F}, \\
& U^{+}=U \vee 0 \text { and } U^{-}=-(U \wedge 0) \text { for } U \in \mathfrak{F} .
\end{aligned}
$$

\section{Zero-sum Games and Variational Inequalities}

Let $\alpha$ be a fixed positive number. Let $F_{1}$ and $F_{2}$ be closed subsets of $X$. Let $f_{1}, f_{2} \in \mathfrak{F} \cap C_{0}(X)$ and put $W=R_{0} g$ for $g \in \mathfrak{F} \cap L^{\infty}(X ; m)$. We assume (A1) and (A2): 
(A1) $X-F_{i}(i=1,2)$ are relatively compact.

(A2) $f_{1} \leqq f_{2} m$-a.e. on $F_{1} \cap F_{2}$.

We call $F_{1}\left(F_{2}\right)$ a stoppable reageon for player I (II resp.). Of courese it is possible to take $F_{1}=F_{2}=X$. Hence we put

$$
\Re=\left\{V \in \mathfrak{F}: f_{1} \leqq V m \text {-a. e. on } F_{1} \text { and } f_{2} \geqq V m \text {-a. e. on } F_{2}\right\} \text {. }
$$

LEMMA 1. There exists a unique solution $U^{\alpha}$ of $\Re$ which satisfies the variational inequality (1.1):

$$
\left(\xi_{\alpha}\left(U^{\alpha}-W, V-U^{\alpha}\right) \geqq 0 \quad \text { for all } V \in \Re .\right.
$$

Proof. Since $\Re$ is a closed convex subset of Hilbert space $\left(\widetilde{F},\left(\mathfrak{E}_{\alpha}\right)\right.$, there exists a unique element $U^{\alpha}$ of $\Re$ such that

$$
\mathfrak{E}_{\alpha}\left(U^{\alpha}-W, U^{\alpha}-W\right) \leqq \xi_{\alpha}(V-W, V-W) \quad \text { for all } V \in \Re .
$$

Since $\Re$ is convex in $\left(\mathfrak{F},\left(\mathfrak{E}_{\alpha}\right),\left(1.1^{\prime}\right)\right.$ is equivalent to $(1.1)$. Therefore we obtain Lemma 1.

Now we note $\Re$ possesses the following property $(R)$ :

(R) $U \vee V \in \Re$ and $U \wedge V \in \Re$ for each $U, V \in \Re$.

LEMMA 2. $U^{\alpha}$ satisfies both inequalities (1.2) and (1.3):

$$
\begin{array}{ll}
\mathfrak{F}_{\alpha}\left(U^{\alpha}-W,\left(V-U^{\alpha}\right)^{+}\right) \geqq 0 & \text { for all } V \in \Re, \\
\mathfrak{F}_{\alpha}\left(U^{\alpha}-W,\left(V-U^{\alpha}\right)^{-}\right) \geqq 0 & \text { for all } V \in \Re .
\end{array}
$$

Proof. We obtain (1.2) ((1.3)), by taking $U^{\alpha} \vee V\left(U^{\alpha} \wedge V\right.$ resp.) instead of $V$ in (1.1) and noting the property (R).

The following lemma is a modification of Lemma 5.1.2 in [5].

LEMMA 3. Let $\left\{U_{n}\right\}_{n}$ be an $\mathfrak{E}_{1}$-Cauchy sequence of quasi-continuous functions in $\tilde{F}$ such that $\left\|U_{n}\right\|_{\infty}$ is bounded uniformly in $n$. Then there exists a subsequence $\left\{U_{n_{k}}\right\}_{k}$ satisfying the condition that for q.e. $x \in X$,

$$
P_{x}\left(\left\{e^{-\alpha t} U_{n_{k}}\left(X_{t}\right)\right\}_{k} \text { converges uniformly in } t \text { on }[0, \infty]\right)=1 \text {. }
$$

Proof. For each $\varepsilon>0$ and $U \in \mathfrak{F}$ we take $T=1 / \alpha \cdot \log \left(U_{\infty} / \varepsilon\right)$. Then for all $t>T$ and a. a. $\omega \in \Omega$ we have

$$
\varepsilon=e^{-\alpha t}: \tilde{U} \|_{\infty} \geqq\left|e^{-\alpha t} \tilde{U}\left(X_{t} \omega\right)\right| .
$$

Hence by noting this fact, in the same line as the proof of Lemma 5.1.2 in [5] we obtain this lemma.

We also need the following lemma and its proof, which are modifications of Theorem 5.2.2 in [5], in order to prove Theorem 1.

LEMMA 4. For each $U \subseteq \mathfrak{F} \cap L^{\infty}(X ; m)$ there exists an additive functienal $A \in \mathfrak{U}_{c}^{x}$ such that for q.e. $x \in X$ and Markov time $\tau$ (1.4) hold:

$$
\left\{\begin{array}{l}
\tilde{U}(x)=E_{x}[A(\infty)] \text { and } \\
\tilde{U}(x)=E_{x}[A(\tau)]+E_{x}\left[e^{-\alpha} \tilde{U}\left(X_{*}\right)\right] .
\end{array}\right.
$$


Proof. We show this lemma, by modifying the proof of Theorem 5.1.1 in [5]. Fix an arbitrary $U \in \mathfrak{F} \cap L^{\infty}(X ; m)$. Then by applying Theorem 4.3 .2 in [5], there exists a quasi-continuous Borel measurable version $\tilde{C}$ of $U$ and a properly exceptional set $N$ such that $\tilde{U}$ vanishes on $N$ for which (1.5) and (1.6) hold:

$$
\begin{aligned}
& n R_{n+\alpha} \tilde{E}(x) \rightarrow \tilde{C}(x) \text { as } n \rightarrow \infty \text { for each } x \in X-N, \\
& n R_{n+\alpha} \tilde{U} \rightarrow \tilde{U} \text { as } n \rightarrow \infty \text { strongly with respect to } \tilde{E}_{1} .
\end{aligned}
$$

For each $n$ we define

$$
g_{n}(x)= \begin{cases}n\left(\tilde{U}(x)-n R_{n+\alpha} \tilde{U}(x)\right) & \text { if } x \in X-N, \\ 0 & \text { if } x \in N .\end{cases}
$$

Then from (1.5), (1.6) and the resolvent equation we obtain $\left(1.5^{\prime}\right)$ and $\left(1.6^{\prime}\right)$ :

$$
R_{\alpha} g_{n}(x) \longrightarrow \tilde{U}(x) \text { as } n \rightarrow \infty \quad \text { for each } x \in X-N,
$$$$
R_{\alpha} g_{n} \longrightarrow \tilde{U} \text { as } n \rightarrow \infty \text { strongly with respect to } \mathfrak{F}_{1} \text {. }
$$

Moreover for each $n$ and $\omega \in \Omega$ we define an additive functional $A_{n}=\left\{A_{n}(t)\right\}_{t \geqq 0} \in \mathfrak{A}_{c}^{\alpha}$ by (1.8) :

$$
A_{n}(t, \omega)=\int_{0}^{t} e^{-\alpha s} g_{n}\left(X_{s} \omega\right) d s \quad \text { for }(t, \omega) \in[0, \infty) \times \Omega
$$

Then we can prove this lemma in similar way to Theorem 5.1.1 of [5].

Let $\mathscr{M}$ be the family of all Markov times. Define an operator $Q_{\tau}$ and subspaces $\mathbb{S}^{+}$and $\mathbb{S}^{-}$by

$$
\begin{aligned}
& Q_{-} k(x)=E_{x}\left[e^{-\alpha=} k\left(X_{-}\right)\right] \quad \text { for } \tau \in \mathscr{M}, x \in X \text { and } k \in \mathfrak{F}, \\
& \mathscr{S}^{+}=\left\{\left(V-U^{\alpha}\right)^{+}: V \in \mathfrak{R}\right\}, \quad \mathbb{S}^{-}=\left\{\left(V-U^{\alpha}\right)^{-}: V \in \mathfrak{R}\right\} .
\end{aligned}
$$

For each Borel subset $B$ of $X, \sigma_{B}\left(\tau_{B}\right)$ expresses the first entry time (the first hitting time resp.) of $B$ :

$$
\sigma_{B}=\inf \left\{t \geqq 0: X_{t} \in B\right\}, \quad \tau_{B}=\inf \left\{t>0: X_{t} \in B\right\} .
$$

THEOREM 1. Uuder (A1) and (A2), there exists a properly exceptional set $N$ for which the solution $U^{\alpha}$ satisfies (1.9) and (1.10):

$$
\begin{array}{ll}
\tilde{U}^{\alpha}-\widetilde{W} \geqq Q_{B_{B_{2}}}\left(\tilde{U}^{\alpha}-\widetilde{W}\right) m \text {-a. e. } & \text { for each } \tau \in \mathscr{M}, \\
\tilde{U}^{\alpha}-\widetilde{W} \leqq Q_{\sigma_{B_{2}} \sigma}\left(\tilde{U}^{\alpha}-\widetilde{W}\right) m \text {-a. e. } & \text { for each } \sigma \in \mathscr{M},
\end{array}
$$

where $B_{i}=\left\{\tilde{U}^{\alpha}=\tilde{f}_{i}\right\} \cap\left(F_{i}-V\right)$ are stopping reageons for $i=1,2$.

Proof. First we show $U^{\alpha} \in \mathfrak{F} \cap L^{\infty}(X ; m)$. Put $a=\left\|f_{1}\right\|_{\infty}+\left\|f_{2}\right\|_{\infty}+\|W\|_{\infty}+1$ and define $U_{a}=\left((W-a) \vee U^{\alpha}\right) \wedge(W+a) \in \Re$. Noting $U_{a}-W=\left((-a) \vee\left(U^{\alpha}-W\right)\right) \wedge a$ are using Markov property of $\left(\mathfrak{F},\left(\xi_{\alpha}\right)\right.$, we obtain

$$
\xi_{\alpha}\left(U_{a}-W, U_{a}-W\right) \leqq \xi_{\alpha}\left(U^{\alpha}-W, U^{\alpha}-W\right) .
$$

By virtue of Lemma 1, we obtain $U^{\alpha} \in \mathfrak{F}$ and $U_{a}=U^{\alpha}-W m$-a. e. This fact shows $U^{\alpha}$ 
$E \tilde{F} L(X ; m)$.

Next for $i=1,2$ and $\varepsilon \geqq 0$ we define

$$
F_{i, \varepsilon}=\left\{x \in X: d(x, y) \leqq \varepsilon \text { for some } y \in F_{i}\right\} .
$$

Then we show that there exists a sequence $\left\{f_{2, n}\right\}_{n \geq 1}$, modifications of $f_{2}$, which satisfies (1.11):

$$
\left\{\begin{array}{l}
f_{2, n} \geqq f_{2} \text { on } X \quad \text { for each } n, \\
f_{2, n}=f_{2} \text { on } F_{2} \quad \text { for each } n \quad \text { and } \\
\tilde{U}^{\alpha} \leqq \tilde{f}_{2, n}-1 / n \text { q. e. on } \overline{X-F_{2,1 / n}} \text { for each } n .
\end{array}\right.
$$

Here $\bar{B}$ denotes the closure of a subset $B$ of $X$. Since $\overline{X-F_{2, \varepsilon}}$ is compact for each $\varepsilon>0$, there exists $W_{\varepsilon} \in C_{0}(X)$ which satisfies $(1.12)$ :

$$
\left\{\begin{array}{l}
W_{\varepsilon} \geqq 0 \quad \text { on } X, \\
W_{\varepsilon}=a \quad \text { on } \overline{X-F_{2, \varepsilon}} \\
\text { and } W_{\varepsilon}=0 \quad \text { on } F_{2},
\end{array}\right.
$$

where $a=\left\|f_{1}\right\|_{\infty}+\left\|f_{2}\right\|_{\infty}+\|W\|_{\infty}+1$. By virtue of Lemma 1.4.2 of [5], for each $\varepsilon>0$ there exists a sequence $\left\{W_{\varepsilon, n}\right\}_{n}$ of $\mathfrak{F} \cap C_{0}(X)$ which satisfies (1.13):

$$
\left\{\begin{array}{l}
\text { Supp }\left[W_{\varepsilon, n}\right] \subset\left\{W_{\varepsilon} \neq 0\right\} \subset X-F_{2} \quad \text { for each } n \\
\text { and }\left\|W_{\varepsilon, n}-W_{\varepsilon}\right\|_{\infty} \leqq 1 / n \quad \text { for each } n .
\end{array}\right.
$$

Hence the subsequence $\left\{W_{1 / n, n}\right\}_{n}$ posesses the property (1.14):

$$
\begin{cases}W_{1 / n, n}=0 \text { on } F_{2} \text { for each } n & \text { and } \\ W_{1 / n, n} \geqq a+1 / n \text { on } X-F_{2,1 / n} & \text { for each } n .\end{cases}
$$

Therefore by putting $f_{2, n}+f_{2}+\left(W_{1,1} \vee W_{1 / 2,2} \vee \cdots \vee W_{1 /(n+1), n+1}\right)^{+} \in \mathfrak{q}$; we have (1.15):

$$
\left\{\begin{array}{l}
f_{2, n} \geqq f_{2} \text { on } X \quad \text { for each } n \\
f_{2, n}=f_{2} \text { on } F_{2} \quad \text { for each } n \text { and } \\
\tilde{f}_{2, n} \geqq \tilde{f}_{2}+a+1 /(n+1) \geqq \tilde{U}^{\alpha}+1 / n m \text {-a.e. } \\
\text { on } X-F_{2,1 /(n+1)} \quad \text { for each } n .
\end{array}\right.
$$

By using Lemma 3.1.4 in [5], we obtain (1.11).

Now we define a sequence $\left\{V_{n}\right\}_{n}$ of $\mathfrak{F}$ by

$$
V_{n}=\left(f_{2, n}-U^{a}\right)^{+} \wedge(1 / n) \quad \text { for } n \geqq 1 .
$$

Then we show the following (1.16):

$$
V_{n} h \in \mathfrak{S}^{+} \text {for each natural number } n \text { and } h \in \mathfrak{F} \text { satisfying } 0 \leqq h \leqq 1 .
$$

Let a natural number $n$ be arbitrary but fixed. Define $V^{\prime}=\left(V_{n} h+U^{\alpha}\right) \wedge f_{2, n}$. Then 
we have

$$
f_{2}=f_{2, n} \geqq V^{\prime} \text { on } F_{2} \text { and } V^{\prime} \geqq U^{\alpha} \wedge f_{2} \geqq f_{1} m \text {-a. e. on } F_{1} .
$$

Therefore $\left(V^{\prime}-U^{\alpha}\right)^{+}=\left(V_{n} h \wedge\left(f_{2, n}-U^{\alpha}\right)\right)^{+}=\left(V_{n} h\right)^{+} \wedge\left(f_{2, n}-U^{\alpha}\right)^{+}=V_{n} h$. Moreover it holds that $V_{n} \subseteq \mathfrak{F} \wedge L^{\infty}(X ; m)$, since $f_{2, n}, U^{\alpha} \in \mathfrak{F}$. Therefore we obtain $V_{n} h \in \mathfrak{F}$ and $V^{\prime} \in \Re$, by applying Theorem 1.4.2 in [5]. Consequently (1.16) holds.

On the other hand we take additive functionals $A, A_{n} \in \mathfrak{A}_{c}^{\alpha} \quad(n \geqq 1)$ and functions $g_{n} \in \mathfrak{F}(n \geqq 1)$, whose definitions are (1.4), (1.8) and (1.7) respectively, corresponding to $U^{\alpha}-W$ instead of $U$. Since $U^{\alpha} \in \Re$, there exists an exceptional set $N_{1}$ satisfying (1.17):

$$
\tilde{f}_{2} \geqq \tilde{U}^{\alpha} \text { on } F_{2}-N_{1} \text { and } \tilde{f}_{1} \leqq \tilde{U}^{\alpha} \text { on } F_{1}-N_{1} .
$$

And we put $B_{2, n}=\left\{\tilde{U}^{a} \geqq \tilde{f}_{2, n}-1 / n\right\} \cap\left(F_{2,1 / n}-N_{1}\right)$ for natural numbers $n$. Hence concerning the expression of $U^{\alpha}-W$ by additive functionals we shall prove (1.18):

$$
\left\{\begin{array}{l}
\text { There exists an } m \text {-negligible Borel subset } N_{2} \text { containing } \\
N_{1} \text {, such that for each } x \in X-N_{2} \text { natural number } l \text { and } \\
\text { Borel subset } B \text { containing } B_{2, l} \text { it holds that } \\
\begin{array}{r}
\tilde{U}^{\alpha}(x)-\widetilde{W}(x)-Q_{\sigma_{B}}\left(\tilde{U}^{\alpha}-\widetilde{W}\right)(x)=\tilde{U}^{\alpha}(x)-\widetilde{W}(x)-Q_{\tau_{B}}\left(\tilde{U}^{\alpha}-\widetilde{W}\right)(x) \\
=E_{x}\left[A\left(\sigma_{B}\right)\right]=E_{x}\left[A\left(\tau_{B}\right)\right] \geqq 0 .
\end{array}
\end{array}\right.
$$

Fix an arbitrary Borel measurable function $h_{0} \in L^{2}(X ; m)$ satisfying $0 \leqq h_{0} \leqq 1$, an arbitrary natural number $l$ and an arbitrary Borel subset $B$ containing $B_{2, l}$. Hence we put

$$
h_{1}=\alpha R_{a} h_{0}-\alpha Q_{\tau_{B}} R_{\alpha} h_{0} .
$$

Since the right hand side of (1.19) equals to $\alpha \cdot E \cdot\left[\int_{0}^{\tau_{B}} e^{-\alpha t} h_{0}\left(x_{t}\right) d t\right]$ and both $R_{\alpha} h_{0}$ and $Q_{\tau_{B}} R_{\alpha} h_{0}$ are $\alpha$-excessive, we have $h_{1} \in \mathscr{V}$ and $0 \leqq h_{1} \leqq 1$. Moreover we put by $\tilde{V}_{l}$ a quasi-continuous Borel version of $V_{l}$. Then from (1.16) and (1.19), we have

$$
\left\{\begin{aligned}
\mathfrak{E}_{\alpha}\left(n G_{n+\alpha}\left(U^{\alpha}-W\right), V_{l} h_{1}\right) & =\mathfrak{E}_{\alpha}\left(\tilde{U}^{\alpha}-\widetilde{W}, n G_{n+\alpha} V_{l} h_{1}\right) \\
& =\mathfrak{F}_{\alpha}\left(n R_{n+\alpha}\left(\tilde{U}^{\alpha}-\widetilde{W}\right), \tilde{V}_{l} h_{1}\right) \\
& =\mathfrak{E}_{\alpha}\left(R_{\alpha} g_{n}, \tilde{V}_{l} h_{1}\right) \\
& =\alpha \cdot\left(g_{n}, \tilde{V}_{l} h_{1}\right) \\
& =\alpha \cdot\left(g_{n} \tilde{V}_{l}, R_{\alpha} h_{0}\right)-\alpha \cdot\left(g_{n} \tilde{V}_{l}, Q_{\tau_{B}} R_{\alpha} h_{0}\right) \\
& =\alpha \cdot\left(R_{\alpha}\left(g_{n} \tilde{V}_{l}\right), h_{0}\right)-\alpha \cdot\left(Q_{\sigma_{B}} R_{\alpha}\left(g_{n} \tilde{V}_{l}\right), h_{0}\right) \text { for each } n .
\end{aligned}\right.
$$

Here we used that $\left\{Q_{\tau_{B}} R_{\alpha}\left(g_{n} \tilde{V}_{l}\right) \neq Q_{\sigma_{B}} R_{\alpha}\left(g_{n} \tilde{V}_{l}\right)\right\}$ is $m$-negligible. Moreover from (1.11) and the definition of $B_{2, l}$, there exists an exceptional set $N_{2}$ containing $N_{1}$ such that for each $s>0$ and $\omega \subseteq \Omega$ satisfying $\sigma<s<\sigma_{B}(\omega)$ we have

$$
X_{s}(\omega) \in\left\{U^{\alpha} \leqq f_{2, l}-1 / l\right\} \cup N_{2} \subset\left\{\tilde{V}_{l}=1 / l\right\} \cup N_{2} .
$$

The equations (1.20), together with this fact, imply 


$$
\begin{aligned}
\left(\tilde{\xi}_{\alpha}\left(n G_{n+\alpha}\left(U^{\alpha}-W\right), V_{l} h_{1}\right)\right. & =\alpha \cdot E_{h_{0} \cdot m}\left[\int_{0}^{\sigma_{B} B} e^{-\alpha_{s}}\left(g_{n} \tilde{V}_{l}\right)\left(X_{s}\right) d s\right] \\
& =\alpha \cdot l^{-1} \cdot E_{h_{0} \cdot m}\left[\int_{0}^{\sigma_{B}} e^{-\alpha s} g_{n}\left(X_{s}\right) d s\right] \\
& =\alpha \cdot l^{-1} \cdot E_{h_{0} \cdot m}\left[A_{n}\left(\sigma_{B}\right)\right] \\
& =\alpha \cdot l^{-1} \cdot E_{h_{0} \cdot m}\left[A_{n}\left(\tau_{B}\right)\right] \quad \text { for each } \mathrm{n} .
\end{aligned}
$$

By considering a subsequence $\left\{n_{k}\right\}$ of $\{n\}$ in the same manner as the proof of Lemma 4 and tending $n_{k}$ infinite, we obtain

$$
\left(\xi_{\alpha}\left(U^{\alpha}-W, V_{l} h_{1}\right)=\alpha \cdot l^{-1} \cdot E_{h_{0} \cdot m}\left[A\left(\sigma_{B}\right)\right]=\alpha \cdot l^{-1} \cdot E_{h_{0} \cdot m}\left[A\left(\tau_{B}\right)\right] .\right.
$$

Consequently we obtain (1.18) from this fact, (1.20), Lemma 2 and Lemma 4.

Next concerning $\alpha$-excessivity of $U^{\alpha}-W$ we shall show (1.21):

$$
\left\{\begin{array}{c}
\tilde{U}^{\alpha}(x)-\widetilde{W}(x) \geqq Q_{\tau \wedge \sigma_{B_{2}, l}}\left(\tilde{U}^{\alpha}-\widetilde{W}\right)(x) \quad \text { for q. e. } x \in X, \\
\text { each } \tau \in \mathscr{M} \text { and each natural number } l .
\end{array}\right.
$$

Fix an arbitrary natural number $l$. Since $Q_{\tau_{B}}\left(\tilde{U}^{\alpha}-\widetilde{W}\right)$ is quasi-continuous for each Borel subset $B$ containing $B_{2, l}$ (see Theorem 4.4.1 in [5]), by applying Lemma 3.1.4 and Theorem 4.2 .1 of [5] to (1.18), there exists a properly exceptional set $N_{3}$ containing $N_{2}$ such that

$$
\left\{\begin{array}{l}
\tilde{U}^{\alpha}(x)-\widetilde{W}(x)-Q_{\tau_{B}}\left(\tilde{U}^{\alpha}-\widetilde{W}\right)(x)=E_{x}\left[A\left(\tau_{B}\right)\right] \geqq 0 \text { for each } x \in X-N_{3} \\
\text { and for each Borel subset } B \text { containing } B_{2, l} .
\end{array}\right.
$$

On the other hand from Theorem 4.3.2 in [5], there exists a properly exceptional set $N_{4}$ for which $\left\{\tilde{U}^{\alpha}<\tilde{f}_{2, l}-1 / l\right\} \cap\left(X-N_{4}\right)$ is finely open and Borel measurable. Hence we put a properly exceptional set $N_{5}=N_{3} \cup N_{4}$ and define a sub-process $\bar{M}=\left\{\bar{X}_{t}\right\}$ by

$$
\bar{X}_{t}(\omega)=\left\{\begin{array}{lll}
x & \text { if } X_{0}(\omega)=x \in N_{5}, \\
X_{t}(\omega) & \text { if } X_{0}(\omega) \notin N_{5} \text { and } t<\sigma_{B_{2, l}}(\omega), \\
\Delta & \text { if } X_{0}(\omega) \notin N_{5} \text { and } t \geqq \sigma_{B_{2, l} l}(\omega),
\end{array}\right.
$$

where $\Delta$ is the death point for $M$. Then $\bar{M}$ becomes to be the standard Markov process in the sense of Blumenthal and Getoor [3] by virtue of Theorems 4.1.3 and 4.1.4 in [5], since $X-N_{5}-B_{2, l}=\left\{\tilde{U}^{\alpha}<\tilde{f}_{2, l}-1 / l\right\} \cap\left(X-F_{2,1 / l}\right) \cap\left(X-N_{5}\right)$ is a finely open Borel set. Now we define

$$
U(x)= \begin{cases}E_{x}\left[A\left(\tau_{B_{2, l}}\right)\right] & \text { if } x \in X-N_{5}-B_{2, l}, \\ 0 & \text { otherwise. }\end{cases}
$$

Then we shall show that $U$ is an $\alpha$-excessive function with respect to the process $\bar{M}$. Because $U$ is nonnegative and Borel measurable. Moreover for each $x \in X-N_{5}$ and each Borel subset $B$ of $X$, 


$$
\begin{aligned}
\bar{Q}_{\bar{\tau}_{B}} U(x) & =E_{x}\left[e^{-\alpha \tau_{B}} U\left(X_{\tau_{B}}\right): \tau_{B}<\sigma_{B_{2, l}}\right] \\
& =E_{x}\left[e^{-\alpha \tau_{B}} E_{X_{\tau_{B}}}\left[A\left(\tau_{B_{2, l}}\right]: \tau_{B}<\sigma_{B_{2, l}}\right]\right. \\
& =E_{x}\left[e^{-\alpha \tau_{B}} A\left(\tau_{B_{2, l}}\right) \circ \theta_{\tau_{B}}: \tau_{B}<\sigma_{B_{2, l}}\right] \\
& =E_{x}\left[A\left(\tau_{B}+\tau_{B_{2, l}} \circ \theta_{\tau_{B}}\right)-A\left(\tau_{B}\right): \tau_{B}<\sigma_{B_{2, l}}\right] \\
& =E_{x}\left[A\left(\tau_{B_{2, l}}\right)-A\left(\tau_{B}\right): \tau_{B}<\tau_{B_{2, l}}\right] \\
& =E_{x}\left[A\left(\tau_{B_{2, l}}\right)-A\left(\tau_{\left(B \cup B_{2, l}\right.}\right)\right] \\
& \leqq E_{x}\left[A\left(\tau_{B_{2, l}}\right)\right]=U(x),
\end{aligned}
$$

where $\bar{Q}$ and $\bar{\tau}_{B}$ imply the transition function function and the hitting time corresponding to the process $\bar{M}$. And while for each $x \in N_{\bar{s}}$ and each Borel subset $B$ of $X$,

$$
\bar{Q}_{\overline{\bar{z}}_{B}} U(x)=\bar{E}_{x}\left[e^{-\alpha \overline{\tau_{B}}} B U\left(X_{\bar{\tau}_{B}}\right)\right]==E_{x}\left[e^{-\alpha \tau_{B}} U(x)\right] \leqq U(x),
$$

where $\bar{E}_{x}$ denotes the expectation starting at $x$ corresponding to $\bar{M}$. Therefore by virtue of Theorem 5.1 in [3, Chapter II] we obtain

$$
U \geqq \beta \bar{R}_{\beta+a} U \text { for each } \beta>0,
$$

where $\left\{\bar{R}_{a}\right\}_{a>0}$ denotes the resolvent corresponding to $\bar{M}$. On the other hand

$$
\bar{Q}_{t} U(x)= \begin{cases}E_{x}\left[A\left(\tau_{B_{2}, l}\right)-A\left(t \wedge \tau_{B_{2, l}}\right)\right] & \text { if } x \in X-N_{5}, \\ \bar{E}_{x}\left[e^{-\alpha t} U(x)\right] & \text { if } x \in N_{5},\end{cases}
$$

which converges to $U(x)$ as $t \downarrow 0$ in both cases. This fact and (1.23) imply that $U$ is $\alpha$-excessive with respect to the process $\bar{M}$ in the sense of Blumenthal and Getoor [3]. By virtue of Proposition 2.8 in [3, Chapeter II], for each $x \in X-N_{\bar{s}}-B_{2, l}$ and $\tau \in \mathscr{M}$

Therefore

$$
\begin{aligned}
E_{x}\left[A\left(\sigma_{B_{2}, l}\right)\right] & =U(x) \geqq \bar{Q}_{\tau} U(x) \\
& =E_{x}\left[e^{-a \tau} U\left(X_{\tau}\right): \tau<\sigma_{B_{2}, l}\right] \\
& =E_{x}\left[e^{-a \tau} E_{x_{\tau}}\left[A\left(\sigma_{B_{2}, l}\right)\right]: \tau<\sigma_{B_{2, l}}\right] \\
& =Q_{\tau \wedge \sigma B_{2, l}}\left(E \cdot\left[A\left(\sigma_{B_{2, l}}\right)\right]\right)(x) .
\end{aligned}
$$

$$
\tilde{U}^{a}-\widetilde{W}-Q_{\sigma_{B_{2}, l}}\left(\tilde{U}^{\alpha}-\widetilde{W}\right) \geqq Q_{-\sigma_{B_{2}, l}}\left[\left(\tilde{U}^{\alpha}-\widetilde{W}\right)-Q_{\sigma_{B_{2, l}}}\left(\tilde{U}^{\alpha}-\widetilde{W}\right)\right] \text { q. e. . }
$$

This shows (1.21).

Finally we shall let $l$ infinite in (1.21). Since $\left\{f_{2, l}\right\}_{l}$ is nondecreasing, $\left\{B_{2, l}\right\}_{l}$ is nonincreasing and $B_{2, l} \supset B_{2}$ for all $l$. Therefore

$$
\lim _{l \rightarrow \infty} \sigma_{B_{2}, l}=\sup _{l} \sigma_{B_{2}, l} \leqq \sigma_{B_{2}} .
$$

Hence we express the left term in (1.24) by $\sigma$. Since $F_{2, n}$ is closed and both $\tilde{U}^{a}$ and $\tilde{f}_{2, l}(l \geqq 1)$ are finely continuous q. e., for any natural numbers $n, l(n \geqq l)$ and any $x \in X-N_{5}$, 


$$
X_{\sigma_{B_{2, n}}} \Subset B_{2, n} \subset B_{2, l} \quad \text { a.s. } P_{x} \text { on }\{\sigma<\infty\} .
$$

By letting $n$ infinite,

$$
X_{\sigma} \Xi B_{2, l} \quad \text { a. s. } P_{x} \text { on }\{\sigma<\infty\} .
$$

Moreover by tending $l$ infinite, for each $x \equiv X-N_{5}$ we obtain

$$
X_{\sigma} \in B_{2} \quad \text { a.s. } P_{x} \text { on }\{\sigma<\infty\},
$$

This, together with (1.24), proves

$$
P_{x}\left(\sigma=\sigma_{B_{2}}\right)=1 \quad \text { for q. e. } x \subseteq X .
$$

Since $\left\{\tilde{U}^{\alpha}-\widetilde{W}>\left\|\tilde{U}^{\alpha}-\widetilde{W}\right\|_{\infty}\right\}$ is exceptional from Lemmas 4.3 .2 and 4.2.4 in [5], by virtue of the bounded convergence theorem and (1.25), (1.21) follows

$$
\left\{\begin{aligned}
\tilde{U}^{\alpha}(x)-\widetilde{W}(x) \geqq Q_{\tau \sim \sigma_{B}}\left(\tilde{U}^{\alpha}-\widetilde{W}\right)(x) \\
\quad \text { for q. e. } x \in X \text { and each } \tau \in \mathscr{M} .
\end{aligned}\right.
$$

This means (1.9). Moreover (1.10) follows in the same line as the proof of (1.9), by changing the signs of (1.9). Consequently Theorem 1 holds.

Now we consider the zero-sum game associated with (1.13) and (1.14). Fix a Borel measurable function $g \in L$ and put $W=R_{\alpha} g$. For $i=1,2$ we define

$$
\mathscr{M}_{i}=\left\{\tau \in \mathscr{M}: P_{x}\left(X_{i} \in F_{i}\right)=1 \text { m-a. a. } x \in X\right\} .
$$

Let $h \in \mathfrak{F} \cap L^{\infty}(X ; m)$. Hence we assume (A3) in addition to (A1) and (A2):

$$
f_{1} \leqq h \leqq f_{2} m \text {-a.e. } \quad \text { on } F_{1} \cap F_{2} \text {. }
$$

For $\tau, \sigma \in \mathscr{M}$ we define a function

$$
J(\tau, \sigma)(x)=E_{x}\left[e^{-a(-\sigma)} L\left(X_{\tau \sigma \sigma}\right)+\int_{0}^{\sigma \sigma} e^{-a t} g\left(X_{t}\right) d t\right],
$$

where

$$
L(\tau, \sigma)= \begin{cases}\tilde{f}_{1}\left(X_{\tau}\right) & \text { on }\{\tau<\sigma\}, \\ \tilde{h}\left(X_{\tau}\right) & \text { on }\{\tau=\sigma\} . \\ \tilde{f}_{2}\left(X_{\sigma}\right) & \text { on }\{\tau>\sigma\} .\end{cases}
$$

Hence we consider the following zero-sum game with stopping times:

$$
\left\{\begin{array}{l}
\text { Find a pair of optimal Markov times }\left(\tau^{*}, \sigma^{*}\right) \\
\in \mathscr{M}_{1} \times \mathscr{M}_{2} \text { attaining an optinal value function } U^{*} \text { such that } \\
\tilde{U}^{*}=\max _{\tau \in \mathscr{M}_{1}} \min _{\sigma \in \mathscr{M}_{2}} J(\tau, \sigma) \\
\quad=\min _{\sigma \in \mathscr{M}_{2}} \max _{\tau \in \mathscr{H}_{1}} J(\tau, \sigma) m \text {-a. e. }
\end{array}\right.
$$

Then the following theorem is derived from Theorem 1.

THEOELEM 2. Under (A1) (A3), $U^{\alpha}$ is an optimal value function and $\left(\sigma_{B_{1}}, \sigma_{B_{2}}\right)$ is a pair of optimal Markov times of the problem (1.27), where $\left.B_{i}=\tilde{U}^{\alpha}=\tilde{f}_{i}\right\} \cap\left(F_{i}-N\right)$ for 
$i=1,2$. Hoerover $\left(\sigma_{B_{1}}, \sigma_{B_{2}}\right)$ is a saddle point of (1.27).

Proof. From (1.27) and the definition of $W$, for $m$-a. a. $x \subseteq X$ and each $\tau \in \mathscr{M}$,

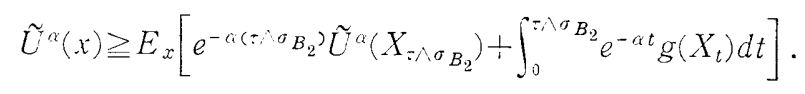

Since $U^{\alpha} \in \Re$, the right term of $(1.28)$ is greater than $J\left(\sigma, \tau_{B_{2}}\right)(x)$. By considering about (1.10) similarly, for each $(\tau, \sigma) \in \mathfrak{M}_{1} \times \mathfrak{M}_{2}$ we obtain

$$
J\left(\tau, \sigma_{B_{2}}\right) \leqq \tilde{U}^{\alpha}=J\left(\sigma_{B_{1}}, \sigma_{B_{2}}\right) \leqq J\left(\tau_{B_{1}}, \sigma\right) \text { m-a. e. }
$$

This completes the proof of Theorem 2 .

\section{Transient Case}

In this section we treat the transient case with both $\alpha=0$ and $W=0$. Let (₹, (æ) be a regular transient Dirichlet space and let $\left(\widetilde{\mho}_{e},(\xi)\right.$ be an extended transient Dirichlet space in the sense of [5]. Let $F_{1}$ and $F_{2}$ be closed subsets of $X$ and let $f_{1}$ and $f_{2}$ be elements of $\mathfrak{F} \cap L^{\infty}(X ; m)$. We assume (A4) in addition to (A1) and (A2):

$$
\left\{f_{2}>f_{1}\right\} \text { is relatively compact. }
$$

Define $\Re_{e}=\left\{V \in \widetilde{F}_{e}: f_{2} \geqq V m\right.$-a.e. on $F_{2}$ and $V \leqq f_{1} m$-a.e. on $\left.F_{1}\right\}$. Then the following lemma holds in similar way to Lemmas 1 and 2 .

LEMMA 5. There exists a unique element $U^{0}$ of $\Re_{e}$ such that

$$
\mathfrak{F}\left(U^{0}, U^{0}\right) \leqq \mathfrak{F}(V, V) \quad \text { for all } V \in \Re_{e} \text {. }
$$

Moreover $U^{0}$ satisfies (2.2) and (2.3):

$$
\begin{gathered}
\left(\xi\left(U^{0}, V-U^{0}\right) \geqq 0 \quad \text { for all } V \in \mathfrak{R}_{e},\right. \\
\left\{\begin{array}{lr}
\left(\mathfrak{E}\left(U^{0},\left(V-U^{0}\right)^{+}\right) \geqq 0\right. & \text { for all } V \in \mathfrak{R}_{e}, \\
\left(\xi\left(U^{0},\left(V-U^{0}\right)^{-}\right) \geqq 0\right. & \text { for all } V \in \Re_{e},
\end{array}\right.
\end{gathered}
$$

The solutions $\left\{U^{\alpha}\right\}_{\alpha>0}$ in Lemma 1 have the following properties.

PROPOSITION 1. $\left\{U^{\alpha}\right\}_{\alpha>0}$ converges to $U^{0}$ in the norm $\mathbb{E}$ as $\alpha \downarrow 0$. Moreover for each $\beta>0,\left\{U^{\alpha+\beta}\right\}_{\alpha>0}$ converges to $U^{\beta}$ as $\alpha \downarrow 0$ in the norm $\mathfrak{E}_{1}$.

PROOF. Define $a=\left\|f_{1}\right\|_{\infty}+\left\|f_{2}\right\|_{\infty}+1$ and $b=\inf _{V \in \Re} \xi(V, V)$. Then

$$
\begin{aligned}
b & =\inf _{V \in \Re} \inf _{\alpha>0} \xi_{\alpha}(V, V) \\
& =\inf _{\alpha>0} \inf _{V \in \Re} \xi_{\alpha}(V, V) \\
& =\lim _{\alpha \downarrow 0} \inf _{V \in \Re}\left(\xi_{\alpha}(V, V)\right. \\
& =\lim _{\alpha \downarrow 0} \xi_{\alpha}\left(U^{\alpha}, U^{\alpha}\right) \\
& =\lim _{\alpha \downarrow 0}\left\{\left(\xi^{\alpha}\left(U^{\alpha}, U^{\alpha}\right)+\alpha\left(U^{\alpha}, U^{\alpha}\right)\right\} .\right.
\end{aligned}
$$

Since $U^{\alpha} \in \Re$ and $\overline{X-F_{i}}(i=1,2)$ are compact, we have 


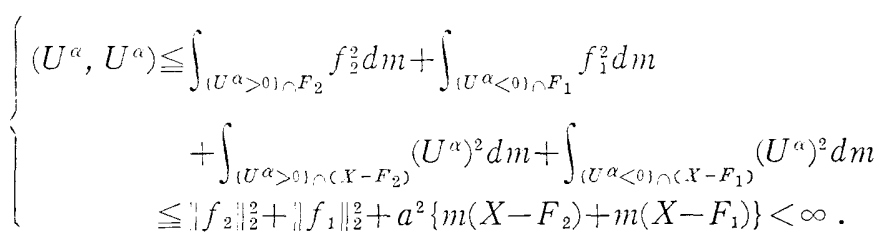

Therefore (2.4), together with this inequality, shows

$$
b=\lim _{a \downarrow 0}\left(\mathfrak{E}\left(U^{\alpha}, U^{\alpha}\right)\right. \text {. }
$$

From Lemma 5 and this fact there exists a subsequence $\left\{U^{a(n)}\right\}_{n}$ of $\left\{U^{\alpha}\right\}_{\alpha>0}$ such that

$$
U^{\alpha(n)} \longrightarrow U^{0} \text { as } n \rightarrow \infty \text { in the norm }(E,
$$

However we can extract a subsequence from any subsequence of $\left\{U^{\alpha}\right\}_{\alpha>0}$ in the same manner, therefore

$$
U^{\alpha} \longrightarrow U^{0} \text { as } \alpha \downarrow 0 \text { in the norm }(\dot{5} \text {. }
$$

Next as for $\left\{U^{\alpha+3}\right\}_{\alpha>0}$ we get the desired property similarly, by replacing $U^{\alpha}, U^{0}$ and (5) with $U^{\alpha+\beta}, U^{\beta}$ and $\xi_{1}$ respectively.

In the transient case the following lemma is trivial but essential for the main theorem.

Lemma 6. Fix an arbitrary Borel subset $B$ of $X$ such that $m(B)<\infty$. Then it holds that

$$
E .\left[\sigma_{(X-B)}\right]<\infty m \text {-a.e. on } X .
$$

Proof. Fix an arbitrary Borel subset $B$ of $X$ such that $m(B)<\infty$. By virtue of Lemma 1.5 .1 in $[5]$, we have

$$
E_{x}\left[\sigma_{(X-B)}\right] \leqq E_{x}\left[\int_{0}^{\infty} I_{B}\left(X_{t}\right) d t\right]<\infty m \text {-a. a. } x \in X,
$$

Here $I_{B}$ denotes the indicator function of $B$. This completes Lemma 6 .

THEOREM 3. Under (A1), (A2) and (A4), there exists a properly exceptional set $N$ for which the solution $U^{0}$ satisfies (2.6) and (2.7):

$$
\begin{aligned}
& \tilde{U}^{0} \geqq P_{\tau \wedge \sigma_{B_{2}}} \tilde{U}^{0} m \text {-a.e. for each } \tau \in \mathcal{M}, \\
& \tilde{U}^{0} \leqq P_{\sigma_{B_{1}} \wedge \sigma} \tilde{U}^{0} \text { m-a.e. for each } \sigma \in \mathcal{M},
\end{aligned}
$$

where $B_{i}=\left\{\tilde{U}^{\alpha}=\tilde{f}_{i}\right\} \cap\left(F_{i}-N\right)$ for $i=1,2$.

Proof. We shall sketch this theorem following the proof of Theorem 1 . We note $U^{0} \in \mathfrak{F}_{e} \cap L^{\infty}(X ; m)$ in the same line as the first part of the proof of Theorem 1 . Since (2.5) holds in the case of $\alpha=0$, we obtain $U^{0} \in \mathfrak{F} \cap L^{\infty}(X ; m)$. Hence we take $F_{i, \varepsilon}(i=$ $1,2 ; \varepsilon \geqq 0)$ and $\left\{f_{2, n}\right\}_{n \geq 1}$ in the same way as the proof of Theorem 1 and put

and

$$
V_{n}=\left(f_{2, n}-U^{0}\right)^{+} \wedge(1 / n) \quad \text { for } n \geqq 1
$$

$$
\mathfrak{G}_{e}^{+}=\left\{\left(V-U^{0}\right)^{+}: \quad V \in \mathfrak{F}_{e}\right\} .
$$

Then in the same line as the proof of (1.16), we obtain 


$$
\left\{\begin{array}{l}
V_{n} h \subseteq \mathfrak{B}_{\bar{\varepsilon}}^{-} \text {for each natural number } n \\
\text { and each } h \in \mho \text { satisfying } 0 \leqq h \leqq 1
\end{array}\right.
$$

Take an exceptional set $N_{1}$ satisfy (2.9):

$$
\tilde{f}_{2} \geqq \tilde{L}^{0} \text { on } F_{2}-N_{1} \text { and } \tilde{f}_{1} \leqq \tilde{L}^{0} \text { on } F_{1}-N_{1} .
$$

And for natural numbers $n$ define

$$
B_{2, n}=\left\{\tilde{U}^{0} \geqq \tilde{f}_{2, n}-1 / n\right\} \frown\left\{F_{2,1 / n}-N_{1}\right) .
$$

Moreover for each $\alpha>0$ we take the additive functional $A^{a} \in \mathfrak{U}_{c}^{\alpha}$, which is defined by (1.4), corresponding to $U^{\alpha}$ instead of $U$. Then in the same line as the proof of Theorem 1 we obtain

$$
\tilde{E}_{\alpha}\left(U^{\alpha}, V_{l} h_{1}\right)=\alpha \cdot l^{-1} \cdot E_{h_{0} \cdot m}\left[A^{\alpha}\left(\sigma_{B}\right)\right]=\alpha \cdot l^{-1} \cdot E_{h_{0} \cdot m}\left[A^{\alpha}\left(\tau_{B}\right)\right],
$$

where $h_{1}$ is defined by (1.19). Hence we have $5\left(U^{0}, V_{l} h_{1}\right) \geqq 0$ and $\left|\left(U^{0}, V_{l} h_{1}\right)\right| \leqq\left|U^{0}\right| i_{\infty} \cdot l^{-1}$. Therefore, from Lemma 4,

$$
\left\{\begin{aligned}
0 & \leqq \lim _{\alpha \downarrow 0} E_{h_{0} \cdot m}\left[A^{\alpha}\left(\sigma_{B}\right)\right]=\lim _{a \downarrow 0} E_{h_{0} \cdot m}\left[A^{\alpha}\left(\tau_{B}\right)\right] \\
& =\lim _{a \downarrow 0}\left\{\left(\tilde{U}^{0}, h_{0}\right)-E_{h_{0} \cdot m}\left[e^{-\alpha \sigma_{B}} \tilde{U}^{0}\left(X_{\sigma_{B}}\right)\right]\right. \\
& =\lim _{\alpha \downarrow 0}\left\{\left(\tilde{U}^{0}, h_{0}\right)-E_{h_{0} \cdot m}\left[e^{-\alpha \varepsilon_{B}} \tilde{U}^{0}\left(X_{\tau_{B}}\right)\right] .\right.
\end{aligned}\right.
$$

While from (A2), (A3) and Lemma 6 we have

$$
E_{h_{0} \cdot m}\left[\sigma_{B}\right]<\infty \text { and } E_{h_{0} \cdot m}\left[\tau_{B}\right]<\infty
$$

for all Borel subsets $B$ containing $B_{2, l}$. Consequently by virtue of the bounded convergence theorem, (2.10) follows

$$
\begin{aligned}
0 & \leqq\left(\tilde{U}^{0}, h_{0}\right)-E_{h_{0} \cdot m}\left[\tilde{U}^{0}\left(X_{\sigma_{B}}\right)\right] \\
& =\left(\tilde{U}^{0}, h_{0}\right)-E_{h_{0} \cdot m}\left[\tilde{U}^{0}\left(X_{\tau_{B}}\right)\right] .
\end{aligned}
$$

Therefore we get Theorem 3 in the same line as the proof of Theorem 1, by using Theorem 5.2 .2 in [5] instead of Lemma 4 and by noting $E_{x}\left[\sigma_{B_{2}}\right]<\infty m$-a. a. $x \in X$.

Now we shall state the zero-sum game associated with (2.1). For $i=1,2$ we define

$$
\mathscr{M}_{f, i}=\left\{\tau \in \mathscr{M}: P_{x}\left(X \in F_{i} \text { and } \tau<\infty\right)=1 \text { m-a. a. } x \in X\right\} .
$$

Let $h \subseteq \mathfrak{F} \cap L^{\infty}(X ; m)$. Hence we assume (A3) in addition to (A1), (A2) and (A4). For $\tau, \sigma \in \mathscr{M}$ satisfying that $P_{y}(\tau<\infty)=P_{y}(\sigma<\infty)=1 m$-a. a. $y \in X$, we define

$$
J^{0}(\tau, \sigma)(x)=E_{x}\left[L\left(X_{\tau \wedge \sigma}\right)\right] \quad \text { for } x \in X,
$$

where $L$ is defined in Section 1. Hence we consider the following problem: 


$$
\begin{aligned}
& \text { Find a pair of optimal stopping times }\left(\tau^{*}, \sigma^{*}\right) \\
& \subseteq \mathscr{M}_{f, 1} \times \mathscr{M}_{f, 2} \text { attaining an optinal value function } U^{*} \text { such that } \\
& \tilde{U}^{*}=\max _{\tau \in \mathscr{H}_{f, 1}} \min _{\sigma \in \mathcal{G}_{f, 2}} J^{0}(\tau, \sigma) \\
& \max _{\sigma \in \mathscr{R}_{j, 2}} \min _{\tau \in \mathscr{S}_{f, 1}} J^{0}(\tau, \sigma) m \text {-a.e. }
\end{aligned}
$$

THEOREM 4. Under (A1) (A4), $U^{0}$ is an optimal value function and $\left(\sigma_{B_{1}}, \sigma_{B_{2}}\right)$ is a pair of optimal stopping times of the problem (2.11), where $B_{i}=\left\{\tilde{U}^{a}=\tilde{f}_{i}\right\} \cap\left(F_{i}-N\right)$ for $i=1,2$. Moreover $\left(\sigma_{B_{1}}, \sigma_{B_{2}}\right)$ is a saddle point of (2.11).

Proof. This proof is in the same line as the proof of Theorem 2.

\section{Examples}

First we consider about Section 1. When the process $M$ is a diffusion, the relation between the variational inequality (1.1) and the game (1.27) is discussed in [1], [4] and [6]. Here we treat the case when $M$ is a jump process. Let $\lambda$ be a positive number and let $q(x, y)$ be a Borel measurable function on $X \times X$, which satisfies (i) $\sim(i i i)$ :

$$
\begin{array}{ll}
\text { (i) } q(x, y)=q(y, x) & \text { for each } x, y \in X, \\
\text { (ii) } q(x, x)=0 & \text { for each } x \in X, \\
\text { (iii) } \int_{X} q(x, y) m(d y)=1 & \text { for each } x \in X .
\end{array}
$$

According to $[3, \mathrm{pp} .63-68]$ we put

$$
P_{t} U(x)=\int_{X} \lambda e^{-\lambda t} q(x, y) U(y) m(d y)
$$

for $t \geqq 0, x \in X$ and $U \in L^{2}$. Then

$$
\left(\xi(U, V)=\int_{Y}(U(x)-U(y))(V(x)-V(y)) \frac{\lambda^{2}}{2} q(x, y) m(d x) m(d y)\right.
$$

for $U, V \in L^{2}$, where $Y=\{(x, y) \in X \times X: x \neq y\}$. From the properties (i) (iii) of $q$ we have

$$
\left(\check{E}(U, U) \leqq 2 \lambda \cdot(U, U)<\infty \quad \text { for } U \in L^{2} .\right.
$$

Therefore from this fact and (1.2.24) in [5] we obtain $\widetilde{\mho}=L^{2}$. On the other hand $\left(\mathfrak{F},\left(\mathfrak{F}_{\alpha}\right)\right.$ is Dirichlet space from Example 1.2.4 in [5]. Hence we can easily check $\left(\mathfrak{F}, \mathfrak{E}_{\alpha}\right)$ is regular, by using $\widetilde{F}=L^{2}$. For a Borel measurable $g \in L^{2}$ we took $W=R_{a} g$ in Section 1. Now we can easily reduce (1.1) to (3.1):

$$
\left\{\begin{array}{c}
\frac{\lambda^{2}}{2} \int_{Y}\left(U^{\alpha}(x)-U^{\alpha}(y)\right)\left(V(x)-U^{\alpha}(x)-V(y)+U^{\alpha}(y)\right) q(x, y) m(d x) m\left(d y^{\prime}\right) \\
\quad+\int_{X}\left(\alpha U^{\alpha}(x)-g(x)\right)\left(V(x)-U^{\alpha}(x)\right) m(d x) \geqq 0 \quad \text { for all } V \in \Re
\end{array}\right.
$$

Next we consider about Section 2. Let $X=D$ be a bounded domain of $R^{n}$ with 
the smooth boundary. Let $m$ be Lebegue ineasure on $R^{n}$. Now we consider $M$ is a diffusion as follows:

$$
\begin{aligned}
& \widetilde{\mho}=H_{0}^{1}(D)=\left\{U \equiv L^{2}(D): \frac{\hat{\partial} U}{\hat{\partial} x_{i}} \in L^{2}(D)(1 \leqq i \leqq n) \text { and } U=0 \text { on } R^{n}-D\right\} \\
& \left(\dot{\xi}(U, V)=\sum_{i, j=1}^{n} \int_{D} \frac{\partial U}{\partial x_{i}} \frac{\hat{o} V}{\partial x_{j}} d x \quad \text { for } U, V \in \widetilde{\mho} .\right.
\end{aligned}
$$

Then $\left(H_{i}(D),\left(\xi_{\alpha}\right)\right.$ is transient from Example 1.5.2 in [5]. Now (2.2) is reduced to (3.2):

$$
\sum_{i, j=1}^{n} \int_{D} \frac{\hat{\partial} U^{\alpha}}{\partial x_{i}}\left(\frac{\partial V}{\partial x_{j}}-\frac{\hat{\partial} U^{\alpha}}{\hat{\partial} x_{j}}\right) d x-\int_{D} g(x)\left(V(x)-U^{\alpha}(x)\right) d x \geqq 0 \quad \text { for all } V \in \Re .
$$

\section{Acknowledgement}

The author would like to thank Prof. N. Furukawa for his helpful advices.

\section{References}

[1] Bensolssan, A. and Friedman, A.: Nonlinear variational inequalities and differential games with stopping times, J. of Func. Analy., 16 (1974), 305-352.

[2] Bismut, J.M.: Sur un probleme de Dynkin, Z. Wahrshein, 39 (1977), 31-53.

[3] Blcienthal, R.M. and Getoor, R.K.: Markov processes and potential theory, Academic press, (1968).

[4] Friedian, A.: Stochastic games and variational inequalities, Arch. Rational Mech. Analy., 51 (1973), 321-346.

[5] Fukushima, M.: Dirichlet forms and Markov processes, North-Holland, Amsterdam, (1980).

[6] Krylov, N.V.: The problem with two free boundaries for an equation and optimal stopping of Markov processes, Dokl. Acad. Nauk USSR 194 (1970), 1263-1265.

[7] NAGAI, H.: On an optimal stopping problem and a variational inequality, J. Math. Soc. Japan, 30(2) (1978), 303-312.

[8] Stettner, L.: Zero-sum Markov games with stopping and impulsive strategies, Applied Math. and Optimi., 8 (1982), 1-24.

[9] ZABCzYк, J.: Stopping games for symmetric Markov processes, Prob. and Math. Stat., 4 (1984), 185-196.

Received October 4, 1988

Communicated by N. Furukawa 